Supporting Information

\title{
Three Dimensionally Free-Formable Graphene Foam with Designed Structures for Energy and Environmental
}

\section{Applications}

\author{
Xi Xu ${ }^{a, b}$, Cao Guan ${ }^{a *}$, Le X $u^{b}$, Yong Hao Tan ${ }^{b, c}$, Danwei Zhang ${ }^{b}$, Yanqing Wang ${ }^{b}$, \\ Hong Zhang ${ }^{b}$, Daniel John Blackwood ${ }^{b}$, John Wang ${ }^{b *}$, Meng Lid ${ }^{d}$, Jun Ding ${ }^{b *}$
}

a Institute of Flexible Electronics, Xi'an Key Laboratory of Flexible Electronics, Northwestern Polytechnical University, Xi'an 710072, China.

b Department of Materials Science and Engineering, Faculty of Engineering, National University of Singapore, Singapore 117575, Singapore.

c NUS Graduate School for Integrative Sciences and Engineering, National University of Singapore, Singapore 119260, Singapore.

d MOE Key Laboratory of Low-grade Energy Utilization Technologies and Systems, CQU-NUS Renewable Energy Materials \& Devices Joint Laboratory, School of Energy \& Power Engineering, Chongqing University, Chongqing 400044, China 
* Corresponding author E-mail: iamcguan@nwpu.edu.cn (Prof. Guan Cao), msewangj@nus.edu.sg (Prof. John Wang), msedingj@nus.edu.sg (Professor Ding)

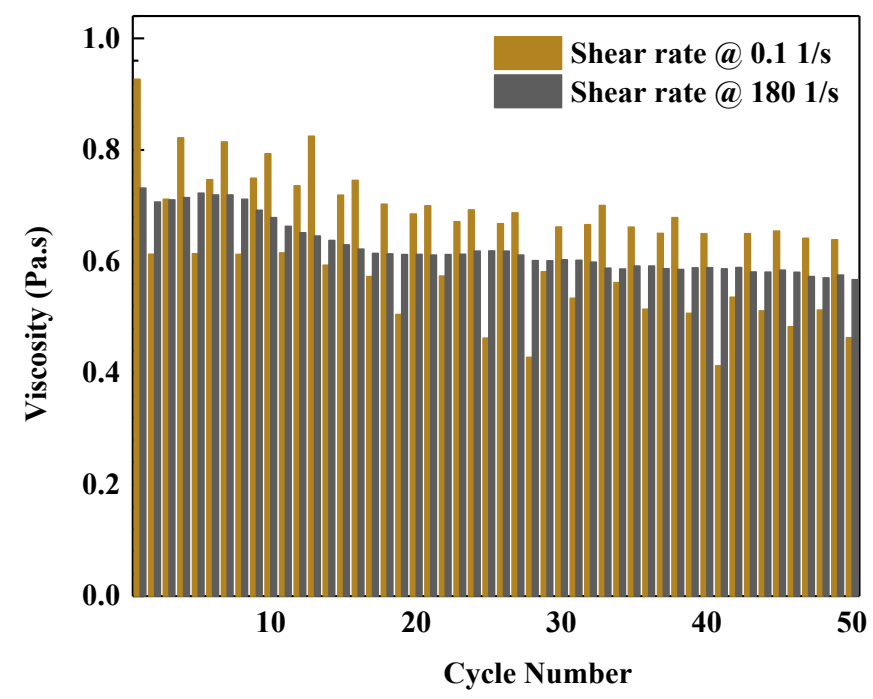

Figure S1. Flow ramp cycling of silica resin (tested for 50 cycles, lasted for 48 hours). 


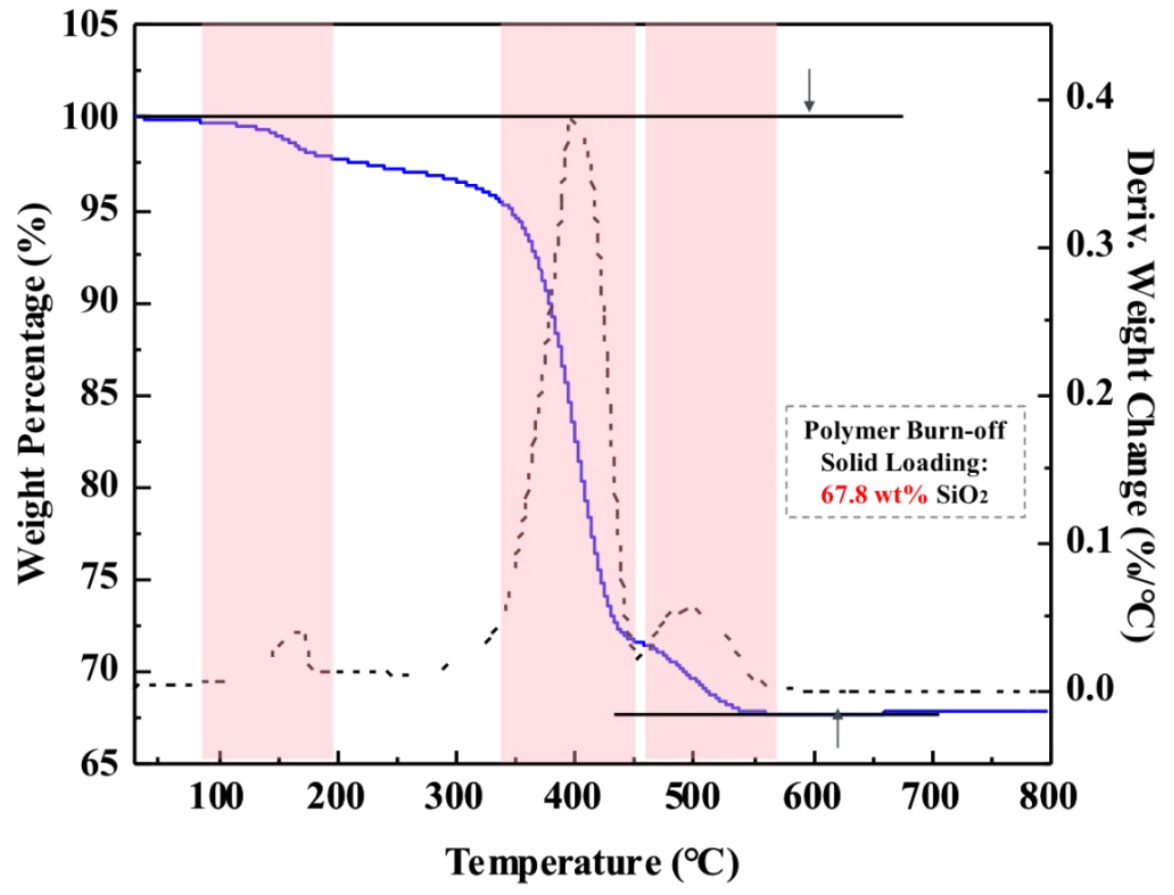

Figure S2. Thermogravimetric Analysis (TGA) graph of silica resin. 


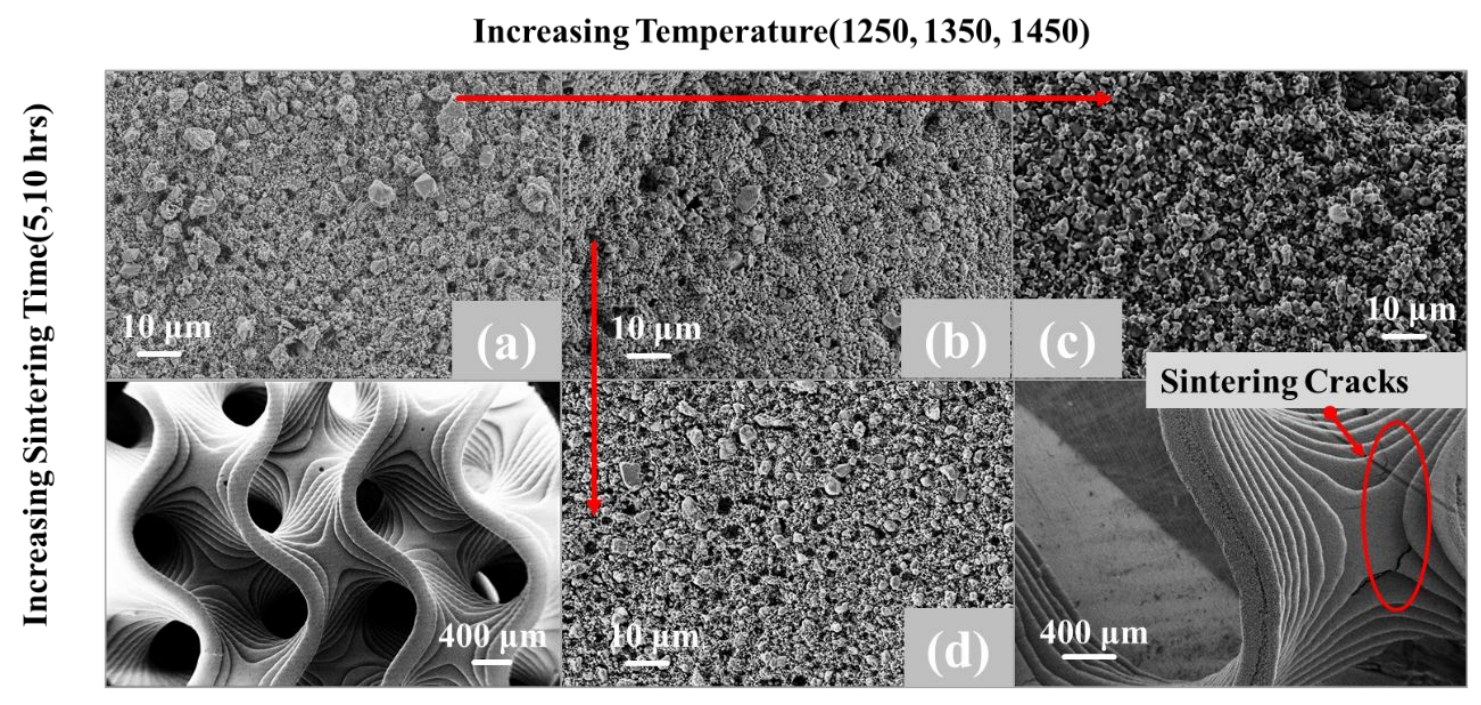

Figure S3. SEM images of silica sintered body under different sintering condition: (a)

dwelling time: 5 hours, sintering temperature: $1250^{\circ} \mathrm{C}$; (b) dwelling time: 5 hours, sintering temperature: $1350^{\circ} \mathrm{C}$; (c) dwelling time: 5 hours, sintering temperature: $1450{ }^{\circ} \mathrm{C}$ (Inserted picture on the bottom right showed sintering cracks under this condition); (d) dwelling time: 10 hours, sintering temperature: $1350{ }^{\circ} \mathrm{C}$. (Inserted picture on the bottom left showed uniform sintering under this condition) 


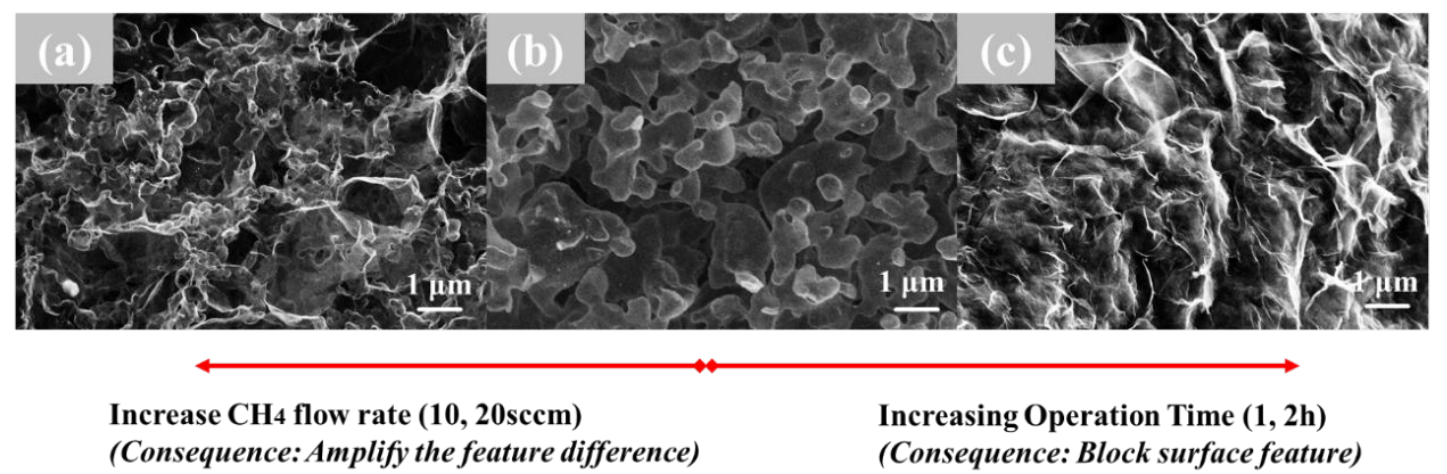

Figure S4. SEM images of 3D graphene foam grown under different flow condition: (a)

$\mathrm{CH}_{4}$ flow rate: $20 \mathrm{sccm}$, operation time: 1 hour; (b) $\mathrm{CH}_{4}$ flow rate: $10 \mathrm{sccm}$, operation time:

1 hours; (c) $\mathrm{CH}_{4}$ flow rate: 20 sccm, operation time: 2 hours. 

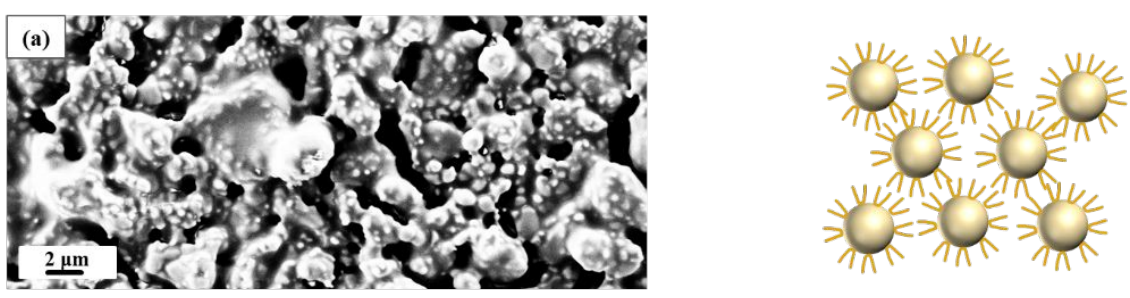

Ligand Removal and Sintering

Colloidal Ceramic Resin

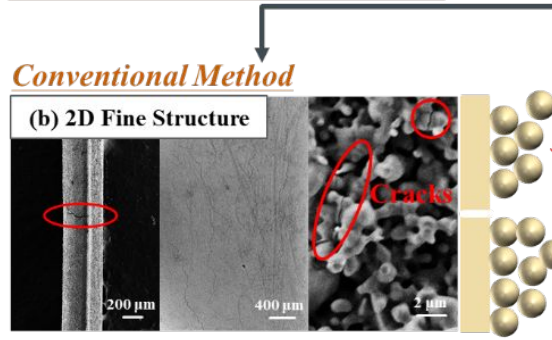

Template Etching

(d) 3D Bulk Structure proly

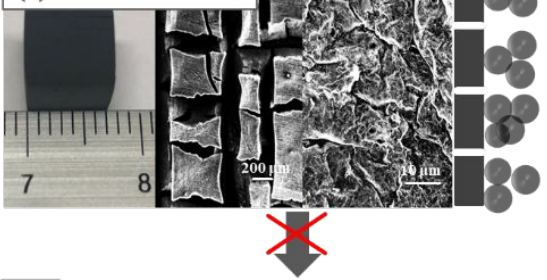

1

Conventional Method

Additive Manufacturing

(c)
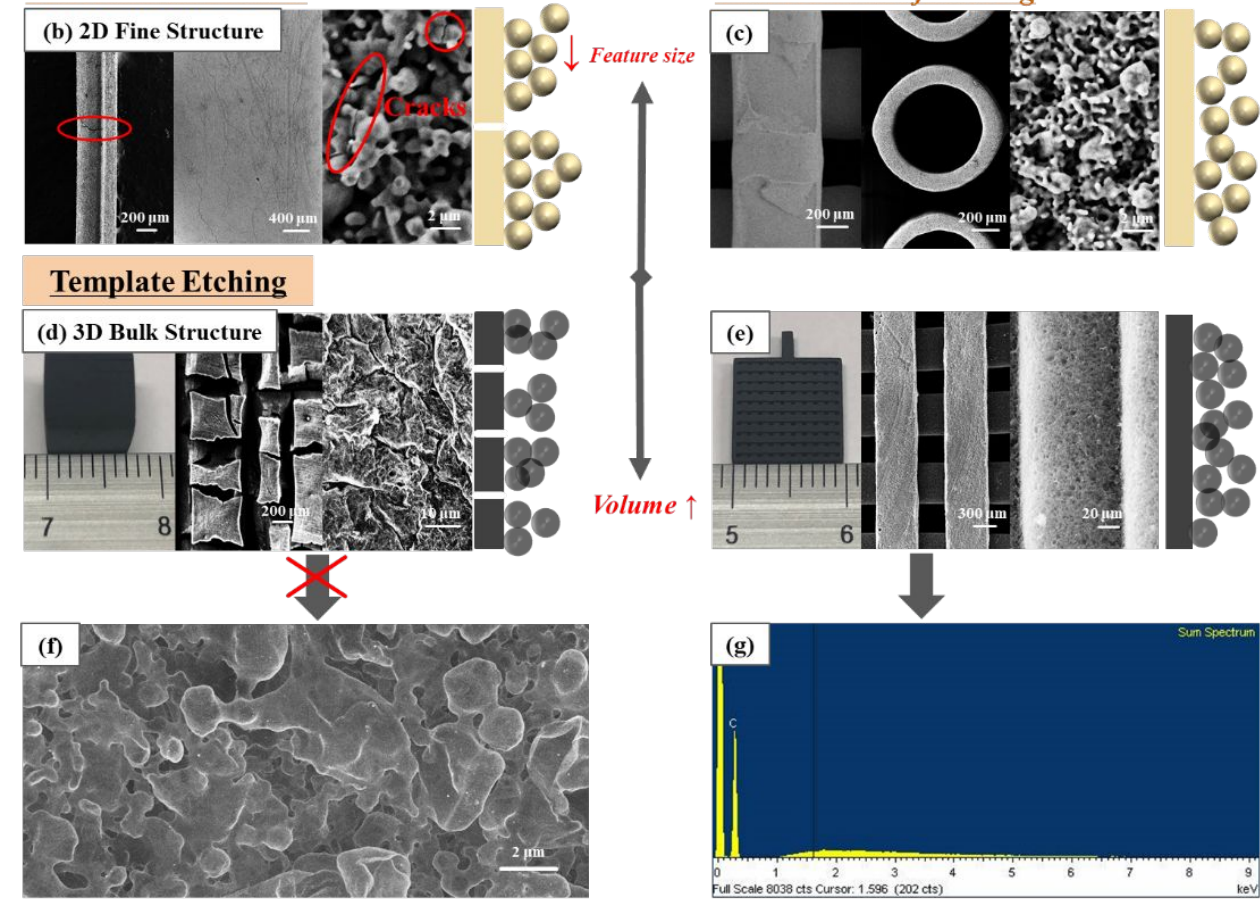

Crack-free phase-pure hierarchical designed graphene foam
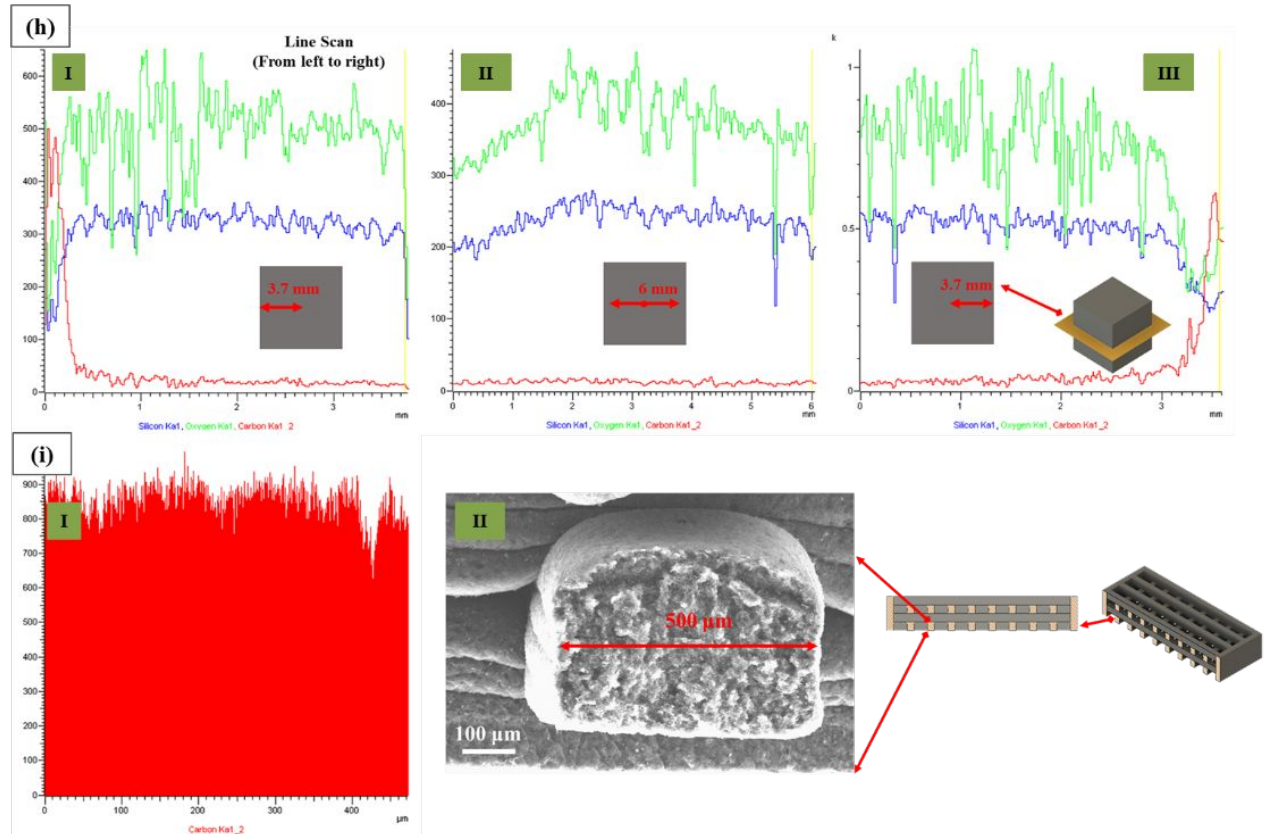
Figure S5. Fabrication of crack-free and phase-pure hierarchical designed graphene foam with controllable grain size and shape. (a) Scanning electron microscopy (SEM) of the colloidal silica resin used in this study; $(b-c)$ Sintering of the spin-coated resin and 3D printed fine structure with same feature size; (d-e) The 3D graphene assemblies from conventional casting(d) and 3D-printed template (e) lead to different product features after wet etching; (f) SEM image of the microstructure of the fully processed 3D graphene foam; (g) Energy-dispersive X-ray spectroscopy (EDX) of the fully processed 3D graphene foam; (h) Cross-sectional elemental line scan of CVD grown solid silica cube $(0.7 \times 0.7 \times$ $0.7 \mathrm{~cm}^{3}$ ) by casting and molding after etching (Direction: from the left (I) to the right (III));

(i) Cross-sectional elemental line scan of CVD grown silica mesh thread (400 $\times 500 \times$ $10000 \mu \mathrm{m}^{3}$ ) by $3 \mathrm{D}$ printing from the left to the right. 


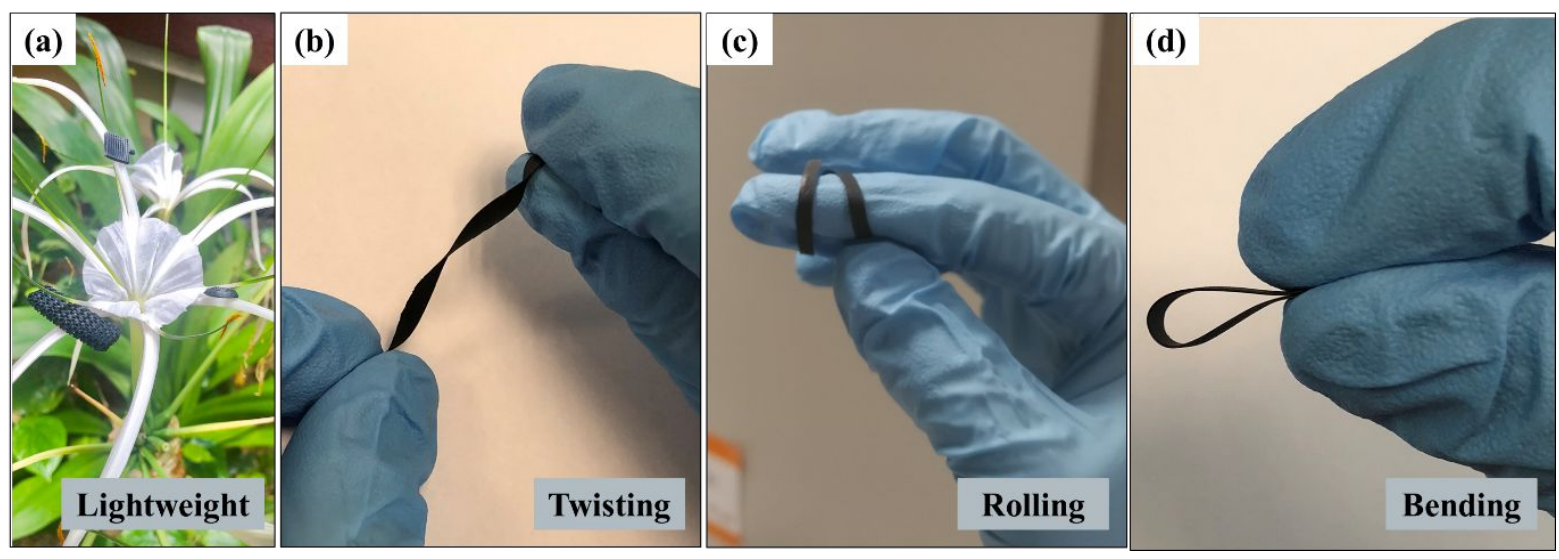

Figure S6. Optical images of as-fabricated 3D lightweight graphene assemblies with various structures (I), showing twisting (II), rolling (III), bending (IV) motion. 


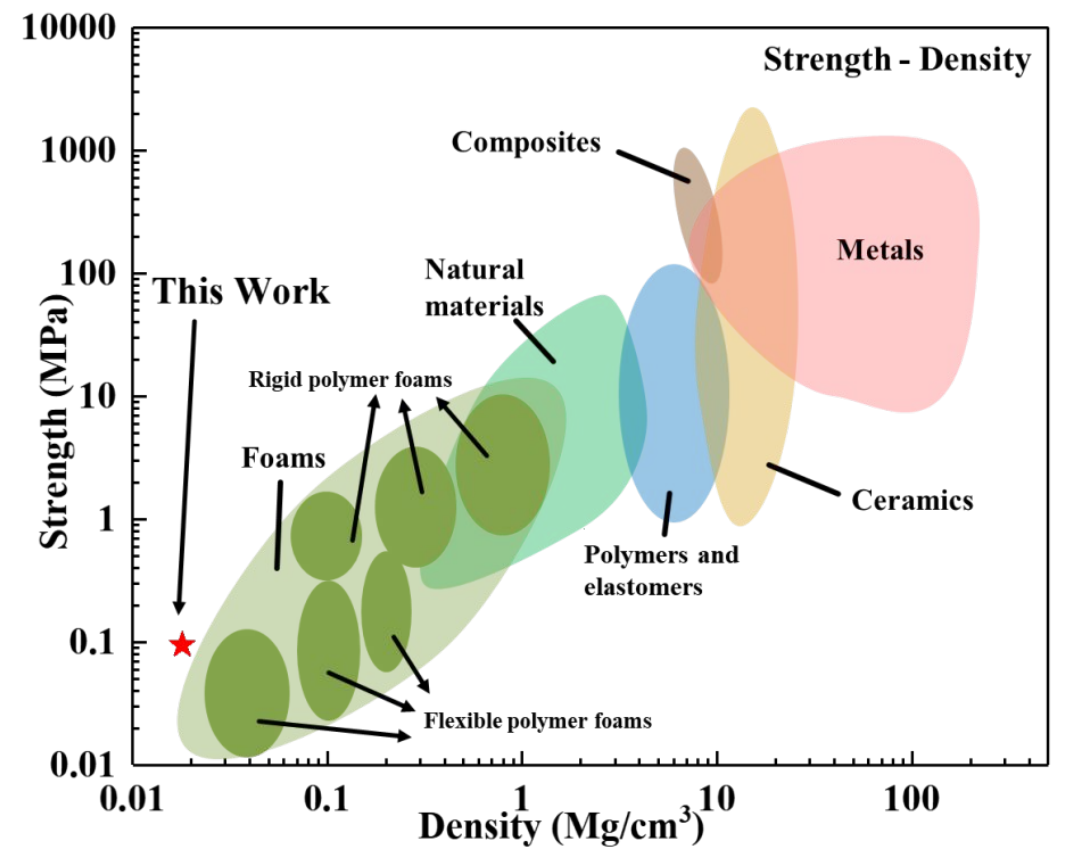

Figure S7. Strength against density in the material property chart. 

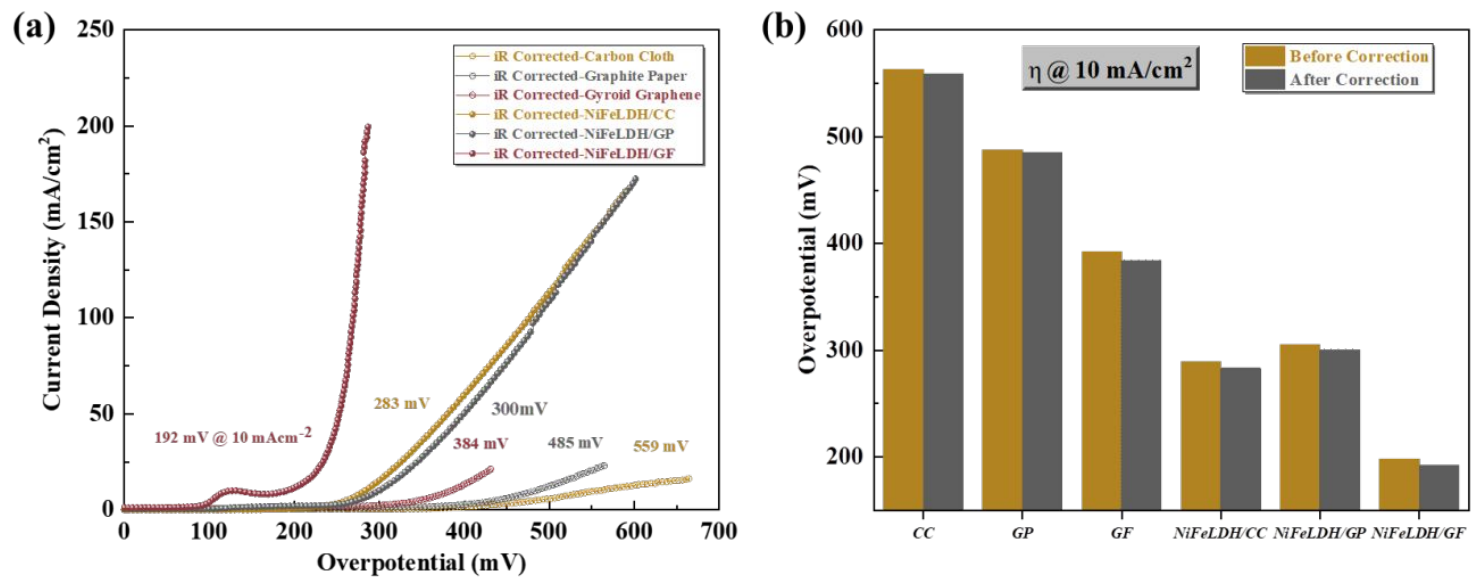

Figure S8. (a) The iR-corrected LSV curves of all samples; (b) overpotential required to drive $10 \mathrm{~mA} / \mathrm{cm} 2$ for all samples before and after iR correction. 


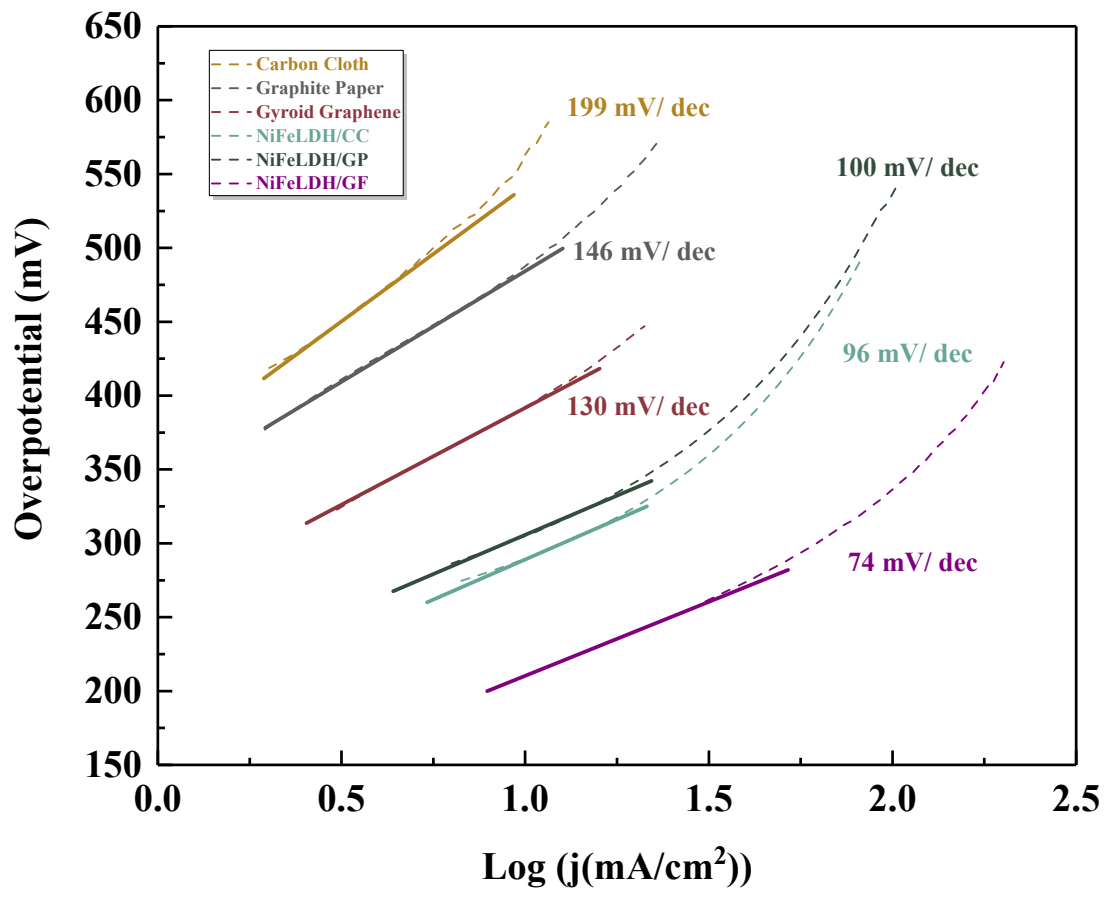

Figure S9. Tafel slope of all samples for OER reaction. 


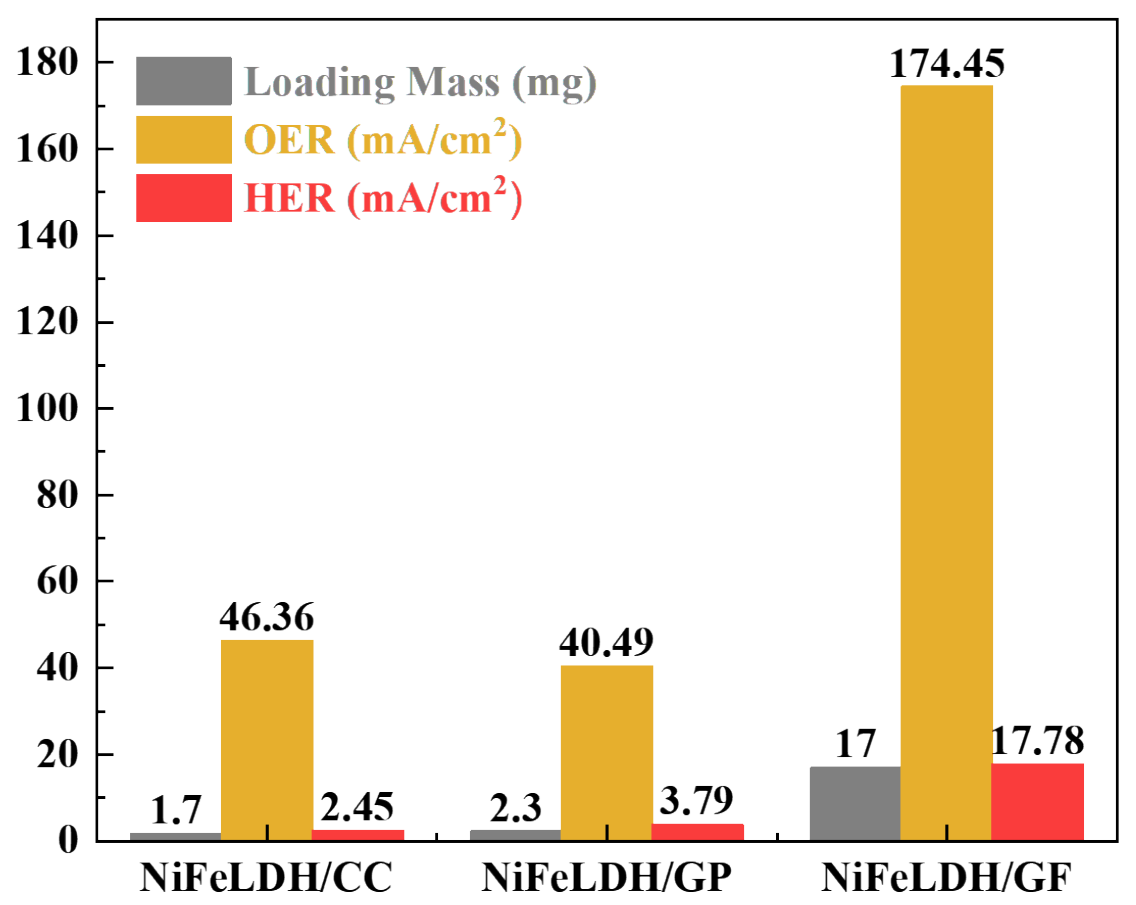

Figure S10. Mass specific current density for NiFeLDH/CC, NiFeLDH/GP, and $\mathrm{NiFeLDH} / \mathrm{GF}$ at $400 \mathrm{mV}$. 

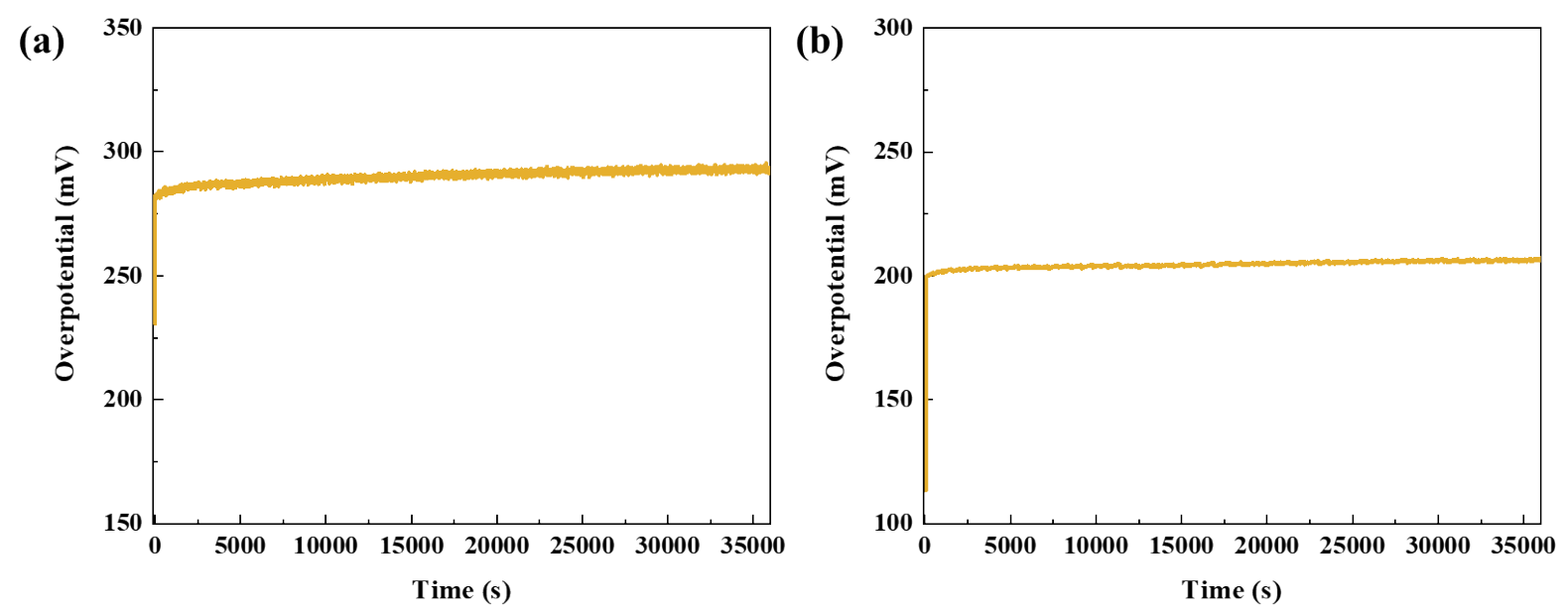

Figure S11. Stability test for NiFe LDH @ GF towards (a) HER; (b) OER at $10 \mathrm{~mA} / \mathrm{cm}^{2}$. 
Table S1. Representative works on fabricating of 3D graphene monolith

\begin{tabular}{|c|c|c|c|c|c|c|}
\hline Sample & Methods & $\begin{array}{l}\text { BET surface } \\
\text { area/ } \mathrm{m}^{2} \mathrm{~g}^{-1}\end{array}$ & $\begin{array}{l}\text { Conductivity/ } \\
\mathrm{S} \mathrm{cm}^{-1}\end{array}$ & $\begin{array}{l}\text { Strength/ } \\
\mathrm{kPa}\end{array}$ & $\begin{array}{l}\text { Compressive } \\
\text { strain }\end{array}$ & $\begin{array}{l}\text { Young's } \\
\text { Modulus/ } \\
\mathrm{kPa}\end{array}$ \\
\hline $\begin{array}{l}\text { 3D-printed gyroid } \\
\text { graphene foam } \\
\text { (This work) }\end{array}$ & $\begin{array}{l}\text { 3D printed silica } \\
\text { derived (DLP) }\end{array}$ & 994.2 & $\begin{array}{c}2.39 \\
\left(\sim 18 \mathrm{mg} / \mathrm{cm}^{3}\right)\end{array}$ & 95 & $55 \%$ & 239.7 \\
\hline $\mathrm{GO}^{1}$ & $\begin{array}{c}\text { 3D printed } \\
\text { (Robocasting; } \\
\text { Areogel) }\end{array}$ & & $\begin{array}{c}0.154 \\
\left(10 \mathrm{mg} / \mathrm{cm}^{3}\right)\end{array}$ & 84.1 & $50 \%$ & 174.6 \\
\hline $\begin{array}{c}\mathrm{GO}+\mathrm{PMA}+ \\
\mathrm{PEG}^{2}\end{array}$ & $\begin{array}{c}\text { 3D printed } \\
\text { (Robocasting; } \\
\text { Areogel) }\end{array}$ & & $\begin{array}{c}0.4 \\
\left(6 \mathrm{mg} / \mathrm{cm}^{3}\right)\end{array}$ & 20 & $50 \%$ & $\begin{array}{c}130 \\
(16 \\
\left.\mathrm{mg} / \mathrm{cm}^{3}\right)\end{array}$ \\
\hline $\mathrm{GO}^{3}$ & $\begin{array}{c}\text { 3D printed } \\
\text { (DLP; Areogel) }\end{array}$ & 130 & $\begin{array}{c}0.64 \\
\left(92 \mathrm{mg} / \mathrm{cm}^{3}\right)\end{array}$ & & & \\
\hline Graphene Paper ${ }^{4}$ & $\begin{array}{l}\text { Glucose-strutted } \\
\text { solution-based } \\
\text { preparation } \\
\text { (Mechanical } \\
\text { compression; } \\
\text { Aerogel) }\end{array}$ & 340 & $\begin{array}{l}5.29 \text { after } \\
\text { reduction } \\
\left(65 \mathrm{mg} / \mathrm{cm}^{3}\right)\end{array}$ & $\begin{array}{l}\text { Foldable } \\
\text { after } \\
\text { compressio } \\
\text { n but brittle }\end{array}$ & & \\
\hline $\begin{array}{l}\text { Graphene } \\
\text { monolith }^{5}\end{array}$ & $\begin{array}{l}\text { Solution-based } \\
\text { self-assembly } \\
\text { preparation }\end{array}$ & 370 & & & & \\
\hline CNT Areogel $^{6}$ & $\begin{array}{l}\text { Solution-based } \\
\text { self-assembly } \\
\text { preparation }\end{array}$ & 580 & $\begin{array}{c}0.26 \\
\left(11.6 \mathrm{mg} / \mathrm{cm}^{3}\right)\end{array}$ & $\begin{array}{c}16.6 \\
(95 \%)\end{array}$ & $33 \%$ & \\
\hline $\begin{array}{l}\text { Porous Carbon } \\
\quad \text { Skelton }^{7}\end{array}$ & $\begin{array}{c}\text { Prolysis of } \\
\text { polymer (silica } \\
\text { powder-assisted) }\end{array}$ & 830 & & & & \\
\hline
\end{tabular}




$\begin{array}{lccc}\begin{array}{l}\text { Graphene } \\ \text { monolith }^{8}\end{array} & \begin{array}{c}\text { Zinc-assisted } \\ \text { solid-state } \\ \text { pyrolysis }\end{array} & 2020 & 1.6\end{array}$


Table S2. Representative works on carbon-materials for desalination

\begin{tabular}{|c|c|c|c|}
\hline Materials & Surface & Power density & Efficiency \\
\hline 3D-printed gyroid graphene foam (This work) & Hydrophobic & 1 & 84.50 \\
\hline 3D-printed treated gyroid graphene foam (This work) & Hydrophilic & 1 & 93.00 \\
\hline Carbonized mushrooms ${ }^{9}$ & Hydrophilic & 1 & 78.00 \\
\hline Vertically aligned graphene sheets membrane ${ }^{10}$ & & 1 & 86.50 \\
\hline Graphene aerogel ${ }^{11}$ & Hydrophilic & 1 & 53.60 \\
\hline $\mathrm{CNT} /$ macroporous silica $^{12}$ & Hydrophobic & 1 & 82.00 \\
\hline $3 \mathrm{D}$-printed porous concave structure ${ }^{13}$ & Hydrophilic & 1 & 85.60 \\
\hline 3D cross-linked honeycomb graphene foam ${ }^{14}$ & Hydrophilic & 1 & 87.00 \\
\hline
\end{tabular}


Table S3. Comparison of electrolysis behaviour with commercial catalysts in alkaline solution after activation.

\begin{tabular}{ccc}
\hline Material & OER & HER \\
& $\left(\eta @ 10 \mathrm{~mA} \mathrm{~cm}^{-2}\right)$ & $\left(\eta @ 10 \mathrm{~mA} \mathrm{~cm}^{-2}\right)$ \\
\hline Graphene Foam (This work) & 392 & 435 \\
NiFe LDH @ GF (This work) & 198 & 230 \\
NiFe LDH @ Nickel Foam ${ }^{15}$ & 240 & 210 \\
3.5 nm Pt @ Nickel Foam ${ }^{15}$ & 340 & 110 \\
RuO $^{16}$ & & - \\
20 wt $\%$ Ir/C $^{17}$ & 350 & - \\
Pt/C - Carbon Fiber ${ }^{18}$ & 280 & 51 \\
\hline
\end{tabular}


Reference:

(1). Zhang, Q.; Zhang, F.; Medarametla, S. P.; Li, H.; Zhou, C.; Lin, D., 3D Printing of Graphene Aerogels. Small 2016, 12, 1702-1708.

(2). García-Tuñon, E.; Barg, S.; Franco, J.; Bell, R.; Eslava, S.; D'Elia, E.; Maher, R. C.; Guitian, F.; Saiz, E., Printing in Three Dimensions with Graphene. Adv. Mater. 2015, 27, 16881693.

(3). Hensleigh, R. M.; Cui, H.; Oakdale, J. S.; Jianchao, C. Y.; Campbell, P. G.; Duoss, E. B.; Spadaccini, C. M.; Zheng, X.; Worsley, M. A., Additive Manufacturing of Complex MicroArchitected Graphene Aerogels. Mater. Horiz. 2018, 5, 1035-1041.

(4). Lee, W. S. V.; Peng, E.; Choy, D. C.; Xue, J. M., Mechanically Robust Glucose Strutted Graphene Aerogel Paper as a Flexible Electrode. J. Mater. Chem. A. 2015, 3, 19144-19147.

(5). Lv, W.; Zhang, C.; Li, Z.; Yang, Q.-H., Self-Assembled 3D Graphene Monolith from Solution. J. Phys. Chem. Lett. 2015, 6, 658-668.

(6). Zou, J.; Liu, J.; Karakoti, A. S.; Kumar, A.; Joung, D.; Li, Q.; Khondaker, S. I.; Seal, S.; Zhai, L., Ultralight Multiwalled Carbon Nanotube Aerogel. ACS Nano 2010, 4, 7293-7302.

(7). Pei, Z.; Li, H.; Huang, Y.; Xue, Q.; Huang, Y.; Zhu, M.; Wang, Z.; Zhi, C., Texturing In Situ: N, S-Enriched Hierarchically Porous Carbon as a Highly Active Reversible Oxygen Electrocatalyst. Energy Environ. Sci. 2017, 10, 742-749.

(8). Jiang, X. F.; Li, R.; Hu, M.; Hu, Z.; Golberg, D.; Bando, Y.; Wang, X. B., Zinc-Tiered Synthesis of 3D Graphene for Monolithic Electrodes. Adv. Mater. 2019, 31, 1901186.

(9). Xu, N.; Hu, X.; Xu, W.; Li, X.; Zhou, L.; Zhu, S.; Zhu, J., Mushrooms as Efficient Solar Steam-Generation Devices. Adv. Mater. 2017, 29, 1606762.

(10). Zhang, P.; Li, J.; Lv, L.; Zhao, Y.; Qu, L., Vertically Aligned Graphene Sheets Membrane 
for Highly Efficient Solar Thermal Generation of Clean Water. ACS Nano 2017, 11, 5087-5093.

(11). Fu, Y.; Wang, G.; Mei, T.; Li, J.; Wang, J.; Wang, X., Accessible Graphene Aerogel for Efficiently Harvesting Solar Energy. ACS Sustainable Chem. Eng. 2017, 5, 4665-4671.

(12). Wang, Y.; Zhang, L.; Wang, P., Self-Floating Carbon Nanotube Membrane on Macroporous Silica Substrate for Highly Efficient Solar-Driven Interfacial Water Evaporation. ACS Sustainable Chem. Eng. 2016, 4, 1223-1230.

(13). Li, Y.; Gao, T.; Yang, Z.; Chen, C.; Luo, W.; Song, J.; Hitz, E.; Jia, C.; Zhou, Y.; Liu, B., 3D-Printed, All-In-One Evaporator for High-Efficiency Solar Steam Generation under 1 Sun Illumination. Adv. Mater. 2017, 29, 1700981.

(14). Yang, Y.; Zhao, R.; Zhang, T.; Zhao, K.; Xiao, P.; Ma, Y.; Ajayan, P. M.; Shi, G.; Chen, Y., Graphene-Based Standalone Solar Energy Converter for Water Desalination and Purification. ACS Nano 2018, 12, 829-835.

(15). Luo, J.; Im, J.-H.; Mayer, M. T.; Schreier, M.; Nazeeruddin, M. K.; Park, N.-G.; Tilley, S. D.; Fan, H. J.; Grätzel, M., Water Photolysis at 12.3\% Efficiency via Perovskite Photovoltaics and Earth-Abundant Catalysts. Science 2014, 345, 1593-1596.

(16). Kou, Z.; Zang, W.; Ma, Y.; Pan, Z.; Mu, S.; Gao, X.; Tang, B.; Xiong, M.; Zhao, X.; Cheetham, A. K., Cage-Confinement Pyrolysis Route to Size-Controlled Molybdenum-Based Oxygen Electrode Catalysts: From Isolated Atoms to Clusters and Nanoparticles. Nano Energy 2019, 104288.

(17). Gong, M.; Li, Y.; Wang, H.; Liang, Y.; Wu, J. Z.; Zhou, J.; Wang, J.; Regier, T.; Wei, F.; Dai, H., An Advanced Ni-Fe Layered Double Hydroxide Electrocatalyst for Water Oxidation. $J$. Am. Chem. Soc. 2013, 135, 8452-8455.

(18). Huang, H.; Yu, C.; Zhao, C.; Han, X.; Yang, J.; Liu, Z.; Li, S.; Zhang, M.; Qiu, J., Iron- 
Tuned Super Nickel Phosphide Microstructures with High Activity for Electrochemical Overall Water Splitting. Nano Energy 2017, 34, 472-480. 